\title{
Giant Nasolabial Cyst Treated Using Neumann Incision: Case Report
}

\author{
Alexandre Beraldo Ordones ${ }^{1}$ Larissa Neri ${ }^{1}$ Ingrid Helena Lopes Oliveira ${ }^{1}$ Miguel Soares Tepedino ${ }^{1}$ \\ Fábio de Rezende Pinna ${ }^{1}$ Richard Louis Voegels ${ }^{1}$
}

${ }^{1}$ Department of ENT, University of Sao Paulo School of Medicine, São Paulo/SP, Brazil

Address for correspondence Alexandre Beraldo Ordones, MD, Rua Dr. Eneas de Carvalho Aguiar, 255/6o andar, 6167, Cerqueira Cesar, São

Int Arch Otorhinolaryngol 2013;17:421-423. Paulo/SP, Brazil (e-mail: ordones.alexandre@gmail.com).

\begin{abstract}
Introduction A nasolabial cyst is an ectodermal development cyst. It presents as a fullness of canine fossa, nasal ala, or vestibule of the nose. It is rare and usually small. Treatment consists of complete surgical excision or transnasal endoscopic marsupialization.

Objective To describe a giant nasolabial cyst case treated using Neumann incision. Case Report A 37-year-old man was referred to the otolaryngology department with

Keywords

- cysts

- nasal obstruction

- nasal cavity nasal obstruction and nasal deformity. Computed tomography showed a nasal cystic lesion $4 \times 4.5 \times 5 \mathrm{~cm}$ wide. Surgical excision using Neumann incision was performed. Discussion Neumann incision provides wide access to the nasal cavity and may be useful in nasolabial cyst treatment.
\end{abstract}

\section{Introduction}

Nasolabial cysts are also known as nasoalveolar cyst or Klestadt cyst and were described in 1882 by Zuckerkandl. ${ }^{1,2}$ They are rare, $^{3}$ affecting 1.6 per 100,000 persons per year. ${ }^{2}$ They occur more frequently in females (4:1), especially among African Americans, in the fourth and fifth decades of life. ${ }^{2,4,5}$ In $90 \%$ of the cases, they are unilateral. ${ }^{5}$ Their growth is slow and painless, so they are often underdiagnosed. ${ }^{1-3,6}$

Patients typically complain of deformity and nasal obstruction. Signs and symptoms include nasal obstruction, local pain, swelling, and facial deformity. ${ }^{1,2,4,7}$ Usually, there is a fullness of the canine fossa, the nasal ala, and nasal vestibule. The bulging reaches the nasal cavity beneath the anterior third of the inferior turbinate, resulting in obliteration of the nasolabial fold and elevation of the alae of the nose. ${ }^{2}$ Because of its close anatomical relation to the nasal cavity and teeth, it may become infected easily. When it is infected, it grows quickly and may be painful. ${ }^{1}$

A nasolabial cyst is diagnosed by clinical examination and is confirmed by histopathologic study. ${ }^{1,2}$ Usually, the nasolabial fold is obliterated. The cysts must be palpated bimanually with one finger in the floor of the nasal vestibule and another in the labial sulcus. Imaging tests, like computed tomography (CT) of the paranasal sinuses and nuclear magnetic resonance (NMR), may be useful. ${ }^{1,8}$ CT shows a cystic lesion located anterior to the piriform aperture; its contents may be homogeneous. Cysts are hyperintense on $\mathrm{T} 1$ and isointense with cerebrospinal fluid on T2-weighted images at NMR, without changes after fat suppression. ${ }^{1,8}$

Differential diagnosis includes cysts of the nasopalatine duct, periapical inflammatory lesions (granuloma, cyst, or abscess), and epidermoid or epidermal inclusion cysts. ${ }^{1}$

Complete surgical excision of the nasolabial cyst is the best treatment. The most used incision is the sublabial. ${ }^{2-4,7}$ Our aim is to describe the Neumann incision to treat a giant nasolabial cyst.

\section{Case Report}

A 37-year-old black man had bilateral nasal obstruction, which improved with saline nasal lavage and nasal corticosteroids. Two years later, he presented with bulging of the nasal vestibule, nasal deformity and asymmetry, and worsened nasal obstruction. Hyposmia, dysgeusia, tenderness, and headache were related. received

May 18, 2012

accepted

August 19, 2012
Copyright @ 2013 by Thieme Publicações DOI http://dx.doi.org/ Ltda, Rio de Janeiro, Brazil $10.1055 / \mathrm{s}-0033-1351674$. ISSN 1809-9777. 
Puncture of the right nasal vestibule was performed, removing $60 \mathrm{~mL}$ of serous liquid, relieving the symptoms. However, the lesion recurred with worsening of symptoms.

CT was performed and a cyst lesion anterior to the right pyriform aperture, $4 \times 4.5 \times 5.5 \mathrm{~cm}$ wide, was found, pushing the nasal septum to left and bulging the palate ( $\mathbf{- F i g . 1} \mathbf{1}$ ).

Complete surgical excision of the cyst was performed using the Neumann incision (-Fig. 1). Histopathologic study revealed squamous and respiratory epithelium with chronic inflammatory process associated with histiocytic reaction. Culture of the secretion isolated Streptococcus viridans.

The patient currently has no evidence of recurrent disease at 2 years postoperatively (-Fig. 2 ).

\section{Discussion}

The origin of nasolabial cysts is controversial. There are two main theories about its growth. The first suggests that the cysts derive from inclusion cysts, secondary to mesenchymal cells after the fusion of medial and lateral nasal prominences to the maxillary prominence during facial skeleton formation. The other theory suggests the cyst is an epithelial remnant of the nasolacrimal duct, running between lateral nasal and maxillary prominences. ${ }^{1,2}$

The most common histologic type is pseudostratified columnar epithelium, followed by squamous stratified epithelium and simple cuboidal epithelium. ${ }^{7} \mathrm{Su}$ et al assessed 10 cysts on electronic microscopy and noticed the cyst had a highly plicated mucosa and were made of nonciliated stratified columnar epithelium, including basal and goblet cells, structurally different from the ciliated columnar epithelium of the paranasal and nasal sinuses and airways. $^{9}$

The treatment of nasolabial cysts consists of complete removal, aiming to prevent infections, define histologic type, and improve esthetics. Fine needle aspiration and cauterization are other treatment options available, but these techniques carry a high recurrence rate. ${ }^{1}$ As reported in the case, cyst puncture for pain relief should always be considered.

Endodontists currently use Neumann incision in performing alveoloplasties. It became popular as an alternative to approaching the maxillary sinus in 1970, replacing CaldwellLuc technique. ${ }^{3}$ It consists of incision in the free edge of the gingiva in the region of the interdental papillae, from the medial portion of the lateral incisor to the lateral portion of the second premolar and first molar, with two vertical extensions, one medial and other lateral to the gingiva and labia, with subsequent elevation of the flap created, allowing full access to the pyriform aperture (-Fig. 2). After the intervention, the mucosal flap is put back into its original position, and the papillae are sutured with absorbable sutures and atraumatic needle, as well as the vertical extensions. The incision considers the vessels and nerves in the region; therefore, the local sensory disturbances such as bleeding are minimal. The potential complications are facial swelling, insensitive gingiva, teeth numbness, and surgical site infection. $^{3}$

The patient must be oriented to blow his or her nose and use a toothbrush on the surgical site. Diet should be reintroduced slowly for the first week. Dental prostheses can be used immediately after the surgery. ${ }^{3}$

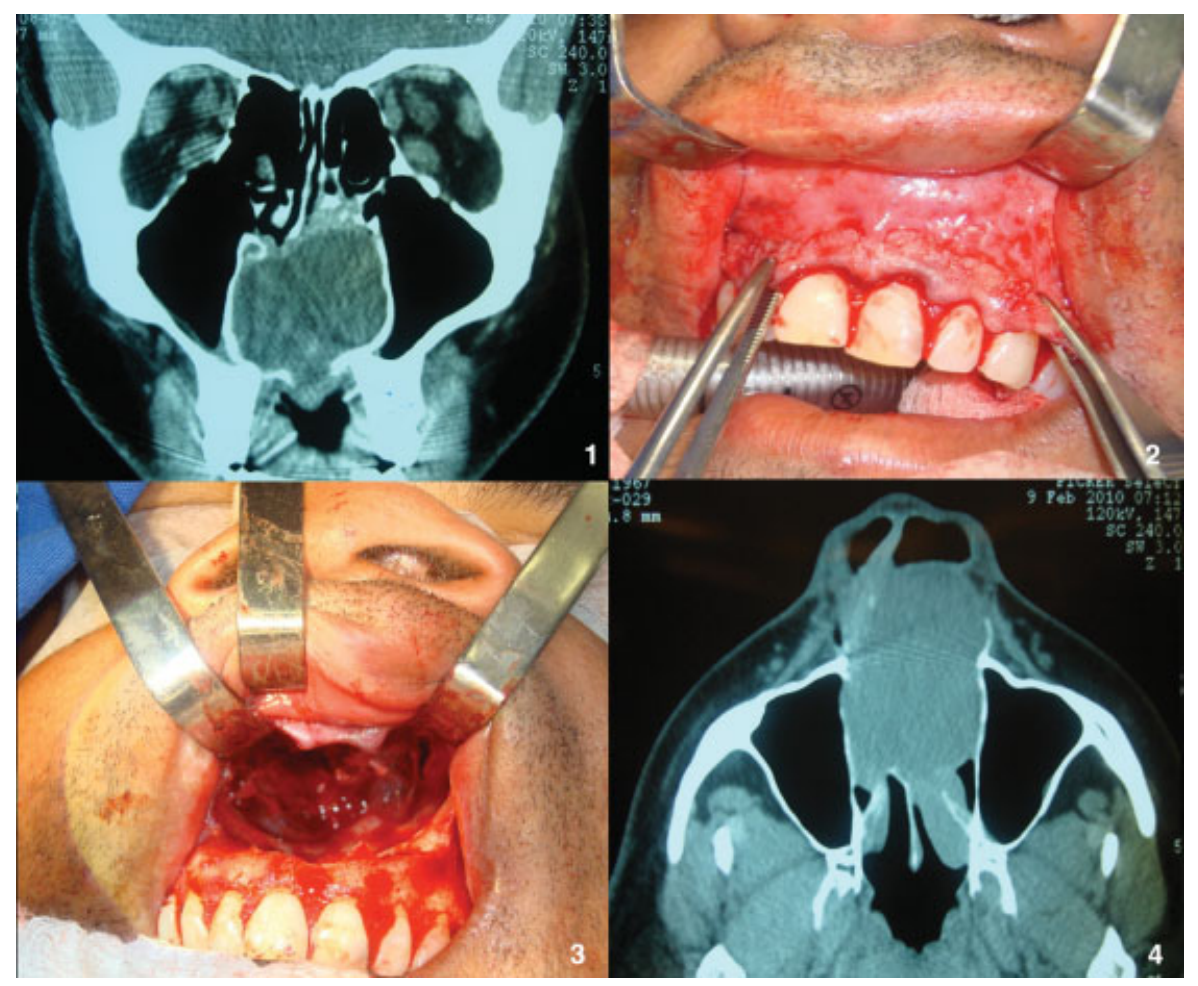

Fig. 1 Computed tomography ( 1 and 4 ) and surgical aspects ( 2 and 3 ). 


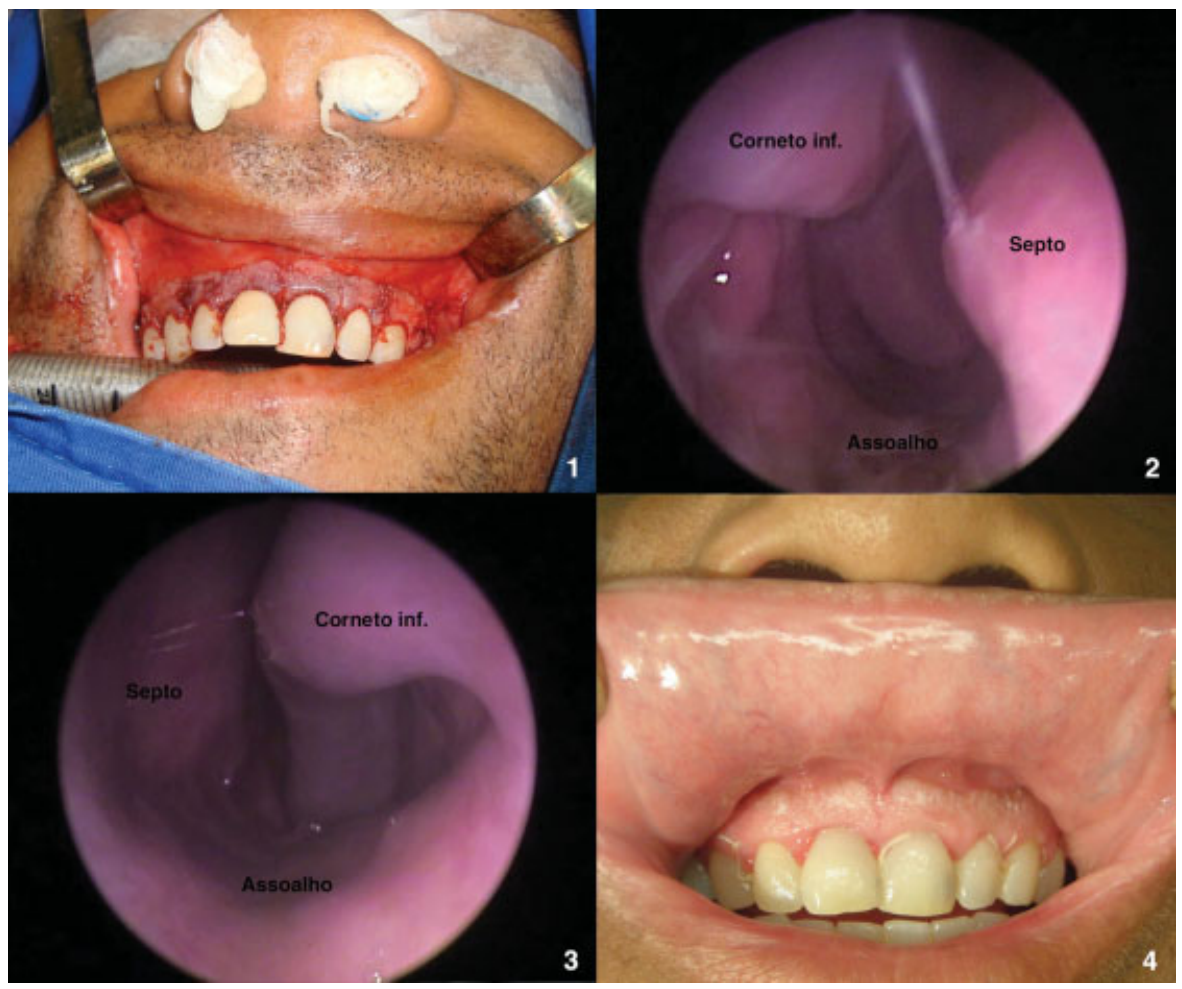

Fig. 2 (1) Postoperative aspect. (2 to 4) Three months after the surgery.

Choi et al described a series of cases in which the cysts varied in size from $1 \times 1 \mathrm{~cm}$ to $3 \times 5 \mathrm{~cm}^{7}$ In the case reported, the cyst measured $4 \times 4.5 \times 5 \mathrm{~cm}$, and during follow-up, no signs of recurrence or complications were noticed.

\section{Conclusion}

Despite its rarity, nasolabial cysts should be considered in differential diagnosis when there is swelling of the floor of the nasal cavity or vestibule. The Neumann incision permits good access to the pyriform aperture and the complete cyst excision.

\section{References}

1 Yuen HW, Julian CY, Samuel CL. Nasolabial cysts: clinical features, diagnosis, and treatment. Br J Oral Maxillofac Surg 2007;45: 293-297

2 el-Din K, el-Hamd AA. Nasolabial cyst: a report of eight cases and a review of the literature. J Laryngol Otol 1999;113:747-749
3 Lee JY, Baek BJ, Byun JY, Chang HS, Lee BD, Kim DW. Comparison of conventional excision via a sublabial approach and transnasal marsupialization for the treatment of nasolabial cysts: a prospective randomized study. Clin Exp Otorhinolaryngol 2009;2: 85-89

4 Marcoviceanu MP, Metzger MC, Deppe H, et al. Report of rare bilateral nasolabial cysts. J Craniomaxillofac Surg 2009;37: 83-86

5 Aquilino RN, Bazzo VJ, Faria RJ, Eid NL, Bóscolo FN. Nasolabial cyst: presentation of a clinical case with CT and MR images. Braz J Otorhinolaryngol 2008;74:467-471

6 Tiago RSL, Maia MS, do Nascimento GMS, Correa JP, Salgado DC. Nasolabial cyst: diagnostic and therapeutical aspects. Braz J Otorhinolaryngol 2008;74:39-43

7 Choi JH, Cho JH, Kang HJ, et al. Nasolabial cyst: a retrospective analysis of 18 cases. Ear Nose Throat J 2002;81:94-96

8 Neves-Pinto RM, Lima PE. Incisão na borda livre da gengiva para o acesso ao seio maxilar: um comentário após oito anos de experiência. Braz J Otorhinolaryngol 1981;47:53-58

9 Su CY, Huang HT, Liu HY, Huang CC, Chien CY. Scanning electron microscopic study of the nasolabial cyst: its clinical and embryological implications. Laryngoscope 2006;116:307-311 